\title{
CHRISTIAN BERG
}

\section{Moment problems and polynomial approximation}

Annales de la faculté des sciences de Toulouse $6^{e}$ série, tome S5 (1996), p. 9-32

<http://www.numdam.org/item?id=AFST_1996_6_S5_9_0>

(C) Université Paul Sabatier, 1996, tous droits réservés.

L'accès aux archives de la revue «Annales de la faculté des sciences de Toulouse » (http://picard.ups-tlse.fr/ annales/) implique l'accord avec les conditions générales d'utilisation (http://www.numdam.org/conditions). Toute utilisation commerciale ou impression systématique est constitutive d'une infraction pénale. Toute copie ou impression de ce fichier doit contenir la présente mention de copyright.

\section{NumDam}

Article numérisé dans le cadre du programme Numérisation de documents anciens mathématiques http://www.numdam.org/ 


\title{
Moment problems and polynomial approximation
}

\author{
Christian Berg(1)
}

\section{Historical introduction}

In Stieltjes famous 1894-memoir $\left[38, \mathrm{n}^{\circ} 24\right]$ he writes:

Nous appellerons problème des moments le problème suivant : trouver une distribution de masse positive sur une droite $(0, \infty)$, les moments d'ordre $k(k=0.1, \ldots)$ étant donnés.

If $\left\{c_{k}\right\}$ denote these numbers, the problem consists in finding a positive measure $\mu$ on $[0, \infty[$ such that

$$
c_{k}=\int_{0}^{\infty} x^{k} \mathrm{~d} \mu(x), \quad k=0,1, \ldots,
$$

and for Stieltjes, a positive measure is given by an increasing function, (cf. $\left.\left[38, \mathrm{n}^{\circ} 37\right]\right)$ :

Le problème des moments que nous avons posé au $\mathrm{n}^{\circ} 24$ nous conduira à considérer une distribution de masse quelconque sur une droite $O x$. Une telle distribution sera parfaitement déterminée si l'on sait calculer la masse totale répandue sur le segment $O x$. Ce sera évidemment une fonction croissante de $x$, et reciproquement, étant donnée une fonction croissante de $x$, on pourra toujours imaginer qu'elle représente, de la manière indiquée, une distribution de masse.

(1) Matematisk Institut, Universitetsparken 5, DK-2100 Copenhagen (Denmark) 


\section{Christian Berg}

Then he defines the integral of a continuous function with respect to an increasing function - the Stieltjes integral.

In present day terminology Stieltjes gave the following characterization of moment sequences.

THEOREM 1.1.- A real sequence $\left\{c_{k}\right\}$ has the form

$$
c_{k}=\int_{0}^{\infty} x^{k} \mathrm{~d} \mu(x), \quad k=0,1, \ldots
$$

for a positive measure $\mu$ with infinite support if and only if

$$
\operatorname{det}\left(c_{i+j}\right)_{0 \leq i, j \leq n}>0, \quad \operatorname{det}\left(c_{i+j+1}\right)_{0 \leq i . j \leq n}>0, \quad \text { for all } n \geq 0
$$

He came to the problem via a study of continued fractions of the form (1.1) below, as we can see in the letter 325 of the Hermite-Stieltjes correspondence, $\mathrm{cf}$. [3] :

\section{5.-Stieltjes a Hermite}

Toulouse, 30 janvier 1892 .

Cher Monsieur,

Je suis [...]. Mais justement, ces jours-ci, j'ai fait sur cette théorie des fractions continues une remarque extrêmement simple et dont $\mathrm{j}$ 'ose vous entretenir. Soit

$$
F=\frac{1}{a_{1} x+\frac{1}{a_{2}+\frac{1}{a_{3} x+\frac{1}{a_{4}+\cdots+\frac{1}{a_{2 n-1} x+\frac{1}{a_{2 n}+\cdots}}}}}}
$$

une fraction continue où

$$
a_{1}, a_{2}, a_{3}, \ldots
$$

sont des nombres réels et positifs. 
Si $x$ est réel et positif, il est clair que les réduites d'ordre pair

$$
\frac{P_{2 n}}{Q_{2 n}}=\frac{1}{a_{1} x+\frac{1}{a_{2}+\cdots+\frac{1}{a_{2 n}}}}
$$

tendent, pour $n=\infty$, vers une limite déterminée $F(x)$. De même les réduites d'ordre impair

$$
\frac{P_{2 n+1}}{Q_{2 n+1}}=\frac{1}{a_{1} x+\frac{1}{a_{2}+\cdots+\frac{1}{a_{2 n+1} x}}}
$$

tendent, pour $n=\infty$, vers une limite déterminée $F_{1}(x)$ et

$$
F_{1}(x)-F(x) \geqq 0 .
$$

[Du reste, d'après un théorème de Stern, on a $F_{1}(x)-F(x)>0$ lorsque la série

$$
a_{1}+a_{2}+a_{3}+a_{4}+\cdots
$$

est convergente; mais, si cette série est divergente, on a

$$
\left.F_{1}(x)=F(x) .\right]
$$

Le théorème général est celui-ci :

Supposons que $x$ ait une valeur réelle ou imaginaire quelconque, en exceptant seulement les valeurs réelles et négatives, en sorte que la partie négative de l'axe des $x$ est considérée comme une coupure. Alors on aura toujours

$$
\lim \frac{P_{2 n}(x)}{Q_{2 n}(x)}=F(x), \quad \lim \frac{P_{2 n+1}(x)}{Q_{2 n+1}(x)}=F_{1}(x) \quad(n=\infty)
$$

et $F(x), F_{1}(x)$ sont des fonctions analytiques, uniformes et sans points singuliers dans le domaine considéré.

Je possède depuis longtemps la démonstration de ce théorème, mais elle est très difficile; [...] 
In the same letter he realizes that a moment problem can have several solutions (if $F$ and $F_{1}$ above are different). He says:

L'existence de ces fonctions $\varphi(u)$ qui, sans être nulles, sont telles que

$$
\int_{0}^{\infty} u^{k} \varphi(u) \mathrm{d} u=0 \quad(k=0,1,2,3, \ldots)
$$

me paraît très remarquable.

Nine months later, in the letters 349 and 351 separated by five days, he gives more details about the convergence of the continued fraction in the two cases: The indeterminate or determinate case depending on whether $\sum a_{n}$ is convergent or divergent:

\section{1.-Stieltjes a Hermite}

Toulouse, 25 octobre 1892 .

Cher Monsieur,

[...] Je me suis proposé d'élucider complètement la nature de cette fraction continue (1.1) dans le cas où les $a_{i}$ sont des nombres réels positifs.

Deux cas sont à distinguer, selon que la série $\sum a_{n}$ est convergente ou divergente. Le premier cas est de beaucoup le plus facile à traiter et, dans ma lettre [(349)], j'ai donné déjà le résultat essentiel; il y a deux limites

$$
\lim \frac{P_{2 n}}{Q_{2 n}}=\frac{p(x)}{q(x)}, \quad \lim \frac{P_{2 n+1}}{Q_{2 n+1}}=\frac{p_{1}(x)}{q_{1}(x)},
$$

où les $p, q, p_{1}, q_{1}$ sont des fonctions holomorphes

$$
p_{1}(x) q(x)-p(x) q_{1}(x)=+1 .
$$

Dans le second cas, où la série $\sum a_{n}$ est divergente, le résultat est aussi simple, mais, pour l'énoncer dans toute sa simplicité, il faut d'abord quelques préliminaires, il est nécessaire d'élargir un peu la notion de l'intégrale définie. En me réservant d'y revenir dans une autre lettre, je me contenterai de dire que la fraction continue est convergente (il n'y 
a pas lieu de distinguer les réduites d'ordre pair et impair) dans tout le plan, excepté la partie négative de l'axe réel. C'est là, en général, une ligne singulière, et il est impossible de continuer la fonction analytique en franchissant cette ligne. Mais ce qui est surtout remarquable c'est la forme analytique sous forme d'intégrale définie qu'on peut donner à cette fonction et dont je parlerai plus loin. [...]

We see here the birth of the Stieltjes integral. Three days earlier Hermite had answered the letter 349 full of admiration:

\section{0.-Hermite a Stieltjes}

Paris, 22 octobre 1892.

\section{Mon Cher Ami,}

Vous êtes un merveilleux géomètre, les recherches nouvelles sur les fractions continues algébriques que vous me communiquez sont un modèle d'invention et d'élégance; ni Gauss. ni Jacobi ne m'ont jamais causé plus de plaisir, et je vous envoie mes vives félicitations, en vous demandant si je dois publier, dans les Comptes rendus, la partie mathématique de votre lettre. [...]

All these results were fully developped in the final memoir [38], where Stieltjes gave several examples of indeterminate measures including the following:

Since

$$
\int_{0}^{\infty} t^{k} \sin (\sqrt[4]{t}) e^{-\sqrt[4]{t}} \mathrm{~d} t=4 \int_{0}^{\infty} u^{4 k+3} \sin (u) e^{-u} \mathrm{~d} u=0, \quad k=0,1, \ldots,
$$

all densities $d_{\lambda}(t)$ on $[0, \infty$ [ given by

$$
d_{\lambda}(t)=e^{-\sqrt[4]{t}}(1+\lambda \sin \sqrt[4]{t}), \quad-1 \leq \lambda \leq 1
$$

have the same moments

$$
c_{k}=\int_{0}^{\infty} t^{k} d_{\lambda}(t) \mathrm{d} t=4(4 k+3) !
$$

Once the moment problem for [0, $[$ [ was formulated and solved by Stieltjes, it was natural to look for a generalization to the whole real line. 
The breakthrough came in a long paper [24] from 1920-21 by Hamburger, who proved:

THEOREM 1.2.- Given $\left\{c_{k}\right\}$, then there exists a positive measure $\mu$ on $\mathbb{R}$ with infinite support such that

$$
c_{k}=\int x^{k} \mathrm{~d} \mu(x), \quad k=0,1, \ldots,
$$

if and only if

$$
\operatorname{det}\left(c_{i+j}\right)_{0 \leq i, j \leq n}>0 \text { for all } n \text {. }
$$

In [33], M. Riesz proved the same result using a technique which eventually was called Hahn-Banach's Theorem. He points out that (1.2) implies that the functional

$$
L(p)=L\left(\sum_{0}^{n} a_{k} t^{k}\right)=\sum_{0}^{n} a_{k} c_{k}
$$

on the polynomials is positive in the following sense:

$$
p(t) \geq 0 \text { for all } t \in \mathbb{R} \Rightarrow L(p) \geq 0 .
$$

Riesz makes an extension of $L$ to a positive linear functional $\tilde{L}$ defined on a space containing the indicator functions for half-lines ] $-\infty, \xi]$, and in this way he constructs the distribution function of the measure $\mu$ to be found.

Riesz says in a footnote to [33]:

J'ai indiqué cette démonstration dans une conférence faite à la Société Mathématique de Stockholm en avril 1918.

The method of Riesz concerning extensions of positive linear functionals on the space of polynomials was taken up by Choquet [22] in an abstract setting called adapted spaces. The method was succesfully applied to the $k$-dimensional moment problem in work of Cassier [19] and Schmüdgen [36].

R. Nevanlinna [31] gave in 1922 another proof of Theorem 1.2 using function theory.

For further details about the historical development of the moment problem see Kjeldsen [28]. 


\section{The Nevanlinna parametrization}

Let $\mathcal{M}^{*}(\mathbb{R})$ denote the set of positive measures on $\mathbb{R}$ with moments of any order and infinite support. For $\mu \in \mathcal{M}^{*}(\mathbb{R})$ let $\left(P_{n}\right)$ be the corresponding orthonormal polynomials determined by

$$
P_{n}(x)=k_{n} x^{n}+l_{n} x^{n-1}+\cdots, \quad k_{n}>0 ; \quad \int P_{n} P_{m} \mathrm{~d} \mu=\delta_{n m} .
$$

In [24] Hamburger proved:

(a) If $\mu$ is determinate, meaning that $\mu$ is the only measure in $\mathcal{M}^{*}(\mathbb{R})$ with the same moments as $\mu$, then $\sum_{n=0}^{\infty}\left|P_{n}(z)\right|^{2}=\infty$ for all $z \in \mathbb{C} \backslash \mathbb{R}$ and all $z \in \mathbb{R}$ except the at most countably many points $z$ where $\mu(\{z\})>0$, in the case of which

$$
\mu(\{z\})=\left(\sum_{n=0}^{\infty} P_{n}^{2}(z)\right)^{-1}
$$

(b) If $\mu$ is indeterminate, meaning that there are several measures in $\mathcal{M}^{*}(\mathbb{R})$ with the same moments as $\mu$, then $\sum_{n=0}^{\infty}\left|P_{n}(z)\right|^{2}<\infty$ for all $z \in \mathbb{C}$.

If $\mu$ is indeterminate we let $V=V_{\mu}$ denote the set of all $\nu \in \mathcal{M}^{*}(\mathbb{R})$ such that

$$
\int x^{n} \mathrm{~d} \mu(x)=\int x^{n} \mathrm{~d} \nu(x) \quad \text { for } n \geq 0 .
$$

Clearly $V$ is a convex set, and it can be proved to be compact in the weak topology, characterized by $\mu_{n} \rightarrow \mu$ weakly if and only if $\int f \mathrm{~d} \mu_{n} \rightarrow \int f \mathrm{~d} \mu$ for all functions $f \in C_{0}(\mathbb{R})$, the continuous functions $f: \mathbb{R}-\mathbb{C}$ vanishing at infinity.

The set $V$ was parametrized by Nevanlinna in [31]. The parameter space is the one-point compactification $\mathcal{P} \cup\{\infty\}$ of the set $\mathcal{P}$ of Pick functions, i.e. the set of holomorphic functions $\varphi: \mathbb{C} \backslash \mathbb{R} \rightarrow \mathbb{C}$ satisfying

$$
\frac{\operatorname{Im} \varphi(z)}{\operatorname{Im} z} \geq 0 \text { for all } z \in \mathbb{C} \backslash \mathbb{R} \text {. }
$$




\section{Christian Berg}

Using the polynomials $\left(Q_{n}\right)$ of the second kind

$$
Q_{n}(x)=\int \frac{P_{n}(x)-P_{n}(y)}{x-y} \mathrm{~d} \mu(y), \quad x \in \mathbb{C},
$$

it is known in the indeterminate case that the series $\sum\left|P_{n}(z)\right|^{2}$ and $\sum\left|Q_{n}(z)\right|^{2}$ converge uniformly on compact subsets of $\mathbb{C}$. Therefore the series

$$
\begin{aligned}
& A(z)=z \sum_{n=0}^{\infty} Q_{n}(0) Q_{n}(z) \\
& B(z)=-1+z \sum_{n=0}^{\infty} Q_{n}(0) P_{n}(z) \\
& C(z)=1+z \sum_{n=0}^{\infty} P_{n}(0) Q_{n}(z) \\
& D(z)=z \sum_{n=0}^{\infty} P_{n}(0) P_{n}(z)
\end{aligned}
$$

determine entire functions, and they satisfy

$$
\operatorname{det}\left(\begin{array}{ll}
A & C \\
B & D
\end{array}\right) \equiv 1
$$

The Nevanlinna parametrization is the homeomorphism $\varphi \mapsto \nu_{\varphi}$ of $\mathcal{P} \cup\{\infty\}$ onto $V$ given by

$$
\int \frac{\mathrm{d} \nu_{\varphi}(x)}{z-x}=\frac{A(z) \varphi(z)-C(z)}{B(z) \varphi(z)-D(z)}, \quad z \in \mathbb{C} \backslash \mathbb{R} .
$$

The special solutions $\nu_{t}, t \in \mathbb{R} \cup\{\infty\}$ corresponding to $\varphi \in \mathcal{P} \cup\{\infty\}$ being a real constant (or $\infty$ ) are called Nevanlinna extremal (short: $N$-extremal). The $N$-extremal solutions form a closed curve in $V$. Since the right-hand side of (2.1) is meromorphic in this case, they are discrete measures of the form

$$
\begin{aligned}
& \nu_{t}=\sum_{\lambda \in \Lambda_{t}} m_{\lambda} \delta_{\lambda}, \quad \Lambda_{t}=\{z \in \mathbb{C} \mid B(z) t-D(z)=0\}, \\
& m_{\lambda}=\frac{A(\lambda) t-C(\lambda)}{B^{\prime}(\lambda) t-D^{\prime}(\lambda)}=\left(\sum_{n=0}^{\infty} P_{n}^{2}(\lambda)\right)^{-1} \text { for } \lambda \in \Lambda_{t}
\end{aligned}
$$

(with $\Lambda_{\infty}=\{z \in \mathbb{C} \mid B(z)=0\}$ ). 
The $N$-extremal solutions were characterized by M. Riesz in 1923 .

Theorem 2.1 ([34]). - Let $\mu \in \mathcal{M}^{*}(\mathbb{R})$.

(a) If $\mu$ is indeterminate and $\nu \in V_{\mu}$, then $\mathbb{C}[t]$ is dense in $L^{2}(\nu)$ if and only if $\nu$ is $N$-extremal.

(b) If $\mu$ is determinate, then $\mathbb{C}[t]$ is dense in $L^{2}(\mu)$.

If $\varphi \in \mathcal{P} \cup\{\infty\}$ is a rational function of degree $n$, then $\nu_{\varphi}$ is called canonical of order $n$ or $n$-canonical. If $\varphi=p / q$, where $p, q$ are polynomials without common zeros, then

$$
\int \frac{\mathrm{d} \nu_{\varphi}(x)}{z-x}=\frac{A(z) p(z)-C(z) q(z)}{B(z) p(z)-D(z) q(z)}
$$

is again meromorphic, showing that $\nu_{\varphi}$ is discrete with masspoints at the zeros of $B p-D q$, the masses being given by the corresponding residues. The 0 -canonical solutions together with $\nu_{\infty}$ are the same as the $N$-extremal solutions.

It is well-known that $\nu \in V$ is $n$-canonical if and only if the measure $\left(1+x^{2}\right)^{-n} \mathrm{~d} \nu(x)$ is N-extremal, cf. [1, p. 121]. Another characterization of $n$-canonical measures is given in the following result of Cassier, which generalizes the Theorem of Riesz.

THEOREM 2.2 ([18], [17]). - A solution $\nu \in V$ to an indeterminate moment problem is $n$-canonical if and only if the closure $\overline{\mathbb{C}[t]}$ of the polynomials in $L^{2}(\nu)$ is of co-dimension $n$.

Theorem 2.1 is the earliest result in the class of problems we shall discuss:

Let $\mu \in \mathcal{M}^{*}(\mathbb{R})$ and $1 \leq \alpha<\infty$. Characterize the $\mu$ and a for which $\mathbb{C}[t]$ is dense in $L^{\alpha}(\mu)$.

This can equally well be studied in $\mathbb{R}^{k}$.

\section{Polynomial approximation}

In $\mathbb{R}^{k}$ we consider the set $\mathcal{M}^{*}\left(\mathbb{R}^{k}\right)$ of positive measures $\mu$ on $\mathbb{R}^{k}$ for which

$$
\int|p(x)| \mathrm{d} \mu(x)<\infty \quad \text { for all } p \in \mathbb{C}[x],
$$

where $\mathbb{C}[x]=\mathbb{C}\left[x_{1}, \ldots, x_{k}\right]$ denotes the set of polynomials in $k$ variables. Let $\mu \in \mathcal{M}^{*}\left(\mathbb{R}^{k}\right)$ and $1 \leq \alpha<\infty$ be given. Our main concern is if $\mathbb{C}[x]$ is dense in $L^{\alpha}(\mu)$. 


\section{Christian Berg}

By Hölder's inequality

$$
\|f\|_{L^{\alpha_{1}(\mu)}} \leq C\left(\alpha_{1}, \alpha_{2}\right)\|f\|_{L^{\alpha_{2}(\mu)}}, \quad 1 \leq \alpha_{1}<\alpha_{2}<\infty,
$$

so there exists a critical exponent $\rho=\rho(\mu) \in[1, \infty]$ such that

(i) $\mathbb{C}[x]$ is dense in $L^{\alpha}(\mu)$ for $1 \leq \alpha<\rho(\mu)$.

(ii) $\mathbb{C}[x]$ is not dense in $L^{\alpha}(\mu)$ for $\rho(\mu)<\alpha<\infty$.

As in section 2 we consider the set $V_{\mu}$ of measures $\nu \in \mathcal{M}^{*}\left(\mathbb{R}^{k}\right)$ such that

$$
\int x^{n} \mathrm{~d} \nu(x)=\int x^{n} \mathrm{~d} \mu(x) \quad \text { for all } n \in \mathbb{N}^{k} .
$$

We say that $\mu$ is determinate if $V_{\mu}=\{\mu\}$, otherwise $\mu$ is indeterminate, in the case of which $V_{\mu}$ is a weakly compact convex set of measures.

We recall the following result of Naimark from 1947:

THEOREM 3.1 ([30])

(i) If $\mu$ is determinate, then $\mathbb{C}[x]$ is dense in $L^{1}(\mu)$.

(ii) If $\mu$ is indeterminate and $\nu \in V_{\mu}$, then $\mathbb{C}[x]$ is dense in $L^{1}(\nu)$ if and only if $\nu$ is an extreme point of $V_{\mu}$.

Proof. - (Gelfand) If $\mathbb{C}[x]$ is not dense in $L^{1}(\mu)$ there exists $f \in L^{\infty}(\mu)$ such that $f \not \equiv 0$ and

$$
\int p(x) f(x) \mathrm{d} \mu(x)=0 \quad \text { for all } p \in \mathbb{C}[x] .
$$

We may assume $f$ real-valued and $-1 \leq f \leq 1$. Then

$$
\mu_{ \pm}=(1 \pm f(x)) \mathrm{d} \mu(x) \in V_{\mu} \quad \text { and } \quad \mu=\frac{1}{2}\left(\mu_{+}+\mu_{-}\right)
$$

with $\mu_{+} \neq \mu_{-}$. This shows that $\mu$ is not an extreme point of $V_{\mu}$.

Conversely, if $\mu=(1 / 2)\left(\mu_{1}+\mu_{2}\right)$ with $\mu_{1}, \mu_{2} \in V_{\mu}$ being different, then since $\mu_{j} \leq 2 \mu$ we have $\mu_{j}=2 f_{j}(x) \mathrm{d} \mu(x)$ with $0 \leq f_{j} \leq 1$, and hence

$$
\int p(x)\left(f_{1}(x)-f_{2}(x)\right) \mathrm{d} \mu(x)=0 \quad \text { for all } p \in \mathbb{C}[x] .
$$

This shows that $\mathbb{C}[x]$ is not dense in $L^{1}(\mu)$. 
TheOREM 3.2.- If $\mu \in \mathcal{M}^{*}\left(\mathbb{R}^{k}\right)$ is indeterminate, then the critical exponent $\rho(\mu) \leq 2$.

Theorem 3.2 was proved in [8] for $k=1$ using the reproducing kernel $\sum P_{n}(x) P_{n}(y)$ for an indeterminate moment problem. The proof in [23] is for any $k$ and uses the theory of operators in Hilbert space.

Theorem 3.3.- For $\mu \in \mathcal{M}^{*}\left(\mathbb{R}^{k}\right)$ let

$$
\lambda_{n}=\int\left(x_{1}^{n}+\cdots+x_{k}^{n}\right) \mathrm{d} \mu(x), \quad n \geq 0 .
$$

If $\sum 1 / \sqrt[2 n]{\lambda_{2 n}}=\infty$, then $\rho(\mu)=\infty$.

For $k=1$ the infinite series condition is the Carleman condition which is sufficient for determinacy. For a proof in this case see [9].

Theorem 3.3 can be deduced from the case $k=1$ using results relating moment problems in one and several dimensions.

Let $\pi_{j}: \mathbb{R}^{k}-\mathbb{R}$ denote the projection $\pi_{j}\left(x_{1}, \ldots, x_{k}\right)=x_{j}$, and for

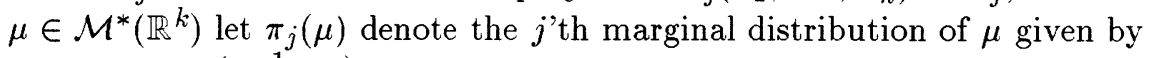
$\pi_{j}(\mu)(B)=\mu\left(\pi_{j}^{-1}(B)\right)$ for Borel sets $B \subseteq \mathbb{R}$.

Theorem $3.4([32])$. - Let $\mu \in \mathcal{M}^{*}\left(\mathbb{R}^{k}\right)$.

(i) If $\mathbb{C}[t]$ is dense in $L^{\alpha}\left(\pi_{j}(\mu)\right), j=1, \ldots, k$ for some $\alpha>1$, then $\mathbb{C}[x]$ is dense in $L^{\beta}(\mu)$ for $1 \leq \beta<\alpha$.

(ii) If $\pi_{1}(\mu), \ldots, \pi_{k}(\mu)$ are determinate. then $\mu$ is determinate.

\section{Remarks}

(a) It is easy to establish (ii) once (i) has been proved. The idea is to prove that any $\nu \in V_{\mu}$ is an extreme point of $V_{\mu}$. If this is so then necessarily $V_{\mu}=\{\mu\}$. For $\nu \in V_{\mu}$ it is clear that $\pi_{j}(\mu)$ and $\pi_{j}(\nu)$ have the same moments so by the assumption in (ii) we have $\pi_{j}(\mu)=\pi_{j}(\nu)$. By the Theorem 2.1 of Riesz $\mathbb{C}[t]$ is dense in $L^{2}\left(\pi_{j}(\nu)\right)$ for $j=1, \ldots, k$ and hence $\rho(\nu) \geq 2$ by (i). By Theorem 3.1 we get that $\nu$ is an extreme point of $\nu_{\mu}$.

(b) There exist determinate measures $\mu \in \mathcal{M}^{*}\left(\mathbb{R}^{k}\right)$ for which $\pi_{1}(\mu), \ldots$, $\pi_{k}(\mu)$ are indeterminate, cf. [32]. One can even obtain this for rotation invariant measures $\mu$, cf. [7].

If $\mu \in \mathcal{M}^{*}(\mathbb{R})$ is determinate we have $\rho(\mu) \geq 2$ by the Theorem 2.1 of Riesz. 


\section{Christian Berg}

THEOREM 3.5. - There exist determinate measures as well as indeterminate measures $\mu \in \mathcal{M}^{*}(\mathbb{R})$ with $\rho(\mu)=2$.

For the proof of this result we need

Lemma 3.6. - Let $\mu \in \mathcal{M}^{*}(\mathbb{R})$ and $x_{1}<\ldots<x_{n}, \alpha_{1}>0, \ldots, \alpha_{n}>0$ be given and define

$$
\sigma=\mu+\sum_{i=1}^{n} \alpha_{i} \delta_{x_{\imath}} .
$$

Then $\rho(\sigma)=\rho(\mu)$.

Proof. - Since $\sigma \geq \mu$ we have $\rho(\mu) \geq \rho(\sigma)$.

Suppose next that $\mathbb{C}(x)$ is non-dense in $L^{a}(\sigma)$ for some $a>1$. We show that $\mathbb{C}[x]$ is non-dense in $L^{\alpha^{\prime}}(\mu)$ for all $\alpha^{\prime}>\alpha$ and therefore $\rho(\mu) \leq \rho(\sigma)$.

If $1 / \alpha+1 / \beta=1$ there exists $f \in L^{\beta}(\sigma) \backslash\{0\}$ such that

$$
\int p(x) f(x) \mathrm{d} \sigma(x)=0 \quad \text { for all } p \in \mathbb{C}[x]
$$

Let $p_{0}(x)=\Pi_{i=1}^{n}\left(x-x_{i}\right)$ and put $g(x)=f(x) p_{0}(x)$. For any $1<\beta^{\prime}<\beta$ we have $g \in L^{\beta^{\prime}}(\mu)$ by Hölders inequality because

$$
\begin{aligned}
\int\left|f(x) p_{0}(x)\right|^{\beta^{\prime}} \mathrm{d} \mu(x) & =\int\left|f(x) p_{0}(x)\right|^{\beta^{\prime}} \mathrm{d} \sigma(x) \\
& \leq c\left(\int|f(x)|^{\beta} \mathrm{d} \sigma(x)\right)^{\beta^{\prime} / \beta}<\infty .
\end{aligned}
$$

Furthermore

$$
\int p(x) g(x) \mathrm{d} \mu(x)=\int p(x) p_{0}(x) f(x) \mathrm{d} \sigma(x)=0 \quad \text { for all } p \in \mathbb{C}[x]
$$

showing that $\mathbb{C}[x]$ is non-dense in $L^{\alpha^{\prime}}(\mu)$, where $1 / \alpha^{\prime}+1 / \beta^{\prime}=1$, i.e. for any $\alpha^{\prime}>a$. 
Let us start with an indeterminate and $N$-extremal measure $\mu$ and let us remove $k \geq 1$ point masses from $\mu$ to get

$$
\tau=\mu-\sum_{a \in \Lambda} \mu(\{a\}) \delta_{a}
$$

where $\Lambda \subseteq \operatorname{supp}(\mu)$ has $k$ points. Then $\tau$ is determinate (and of index of determinacy $k-1$, cf. sect. 5). If we instead add $k$ new masses to $\mu$, we get

$$
\sigma=\mu+\sum_{i=1}^{k} \alpha_{i} \delta_{a_{\imath}}, \quad \alpha_{i}>0
$$

where $a_{1}, \ldots, a_{k}$ are different points of $\mathbb{R} \backslash \operatorname{supp}(\mu)$. Then $\sigma$ is indeterminate and $k$-canonical, cf. [18], [17].

By the Lemma 3.6

$$
\rho(\mu)=\rho(\sigma)=\rho(\tau)=2
$$

since $\rho(\tau) \geq 2$ by Theorem 2.1 and $\rho(\sigma) \leq 2$ by Theorem 3.2.

The denseness of $\mathbb{C}[t]$ in $L^{\alpha}(\mu)$ is governed by a function $M_{\alpha}$ introduced in connection with the Bernstein problem of weighted approximation, $\mathrm{cf}$. [29]. This function is called the Hall-Mergelyan majorant.

Lemma 3.7.- Let $\mu \in \mathcal{M}^{*}(\mathbb{R})$ and $1 \leq \alpha<\infty$ and define

$$
M_{\alpha}(z)=\sup \left\{|p(z)| \mid \int \frac{|p(t)|^{\alpha}}{(1+|t|)^{\alpha}} \mathrm{d} \mu(t) \leq 1, p \in \mathbb{C}[t]\right\} .
$$

Then $\mathbb{C}[t]$ is dense in $L^{\alpha}(\mu)$ if and only if there exists $z_{0} \in \mathbb{C} \backslash \operatorname{supp}(\mu)$ such that $M_{\alpha}\left(z_{0}\right)=\infty$. In the affirmative case $M_{\alpha}(z)=\infty$ for all $z \in \mathbb{C} \backslash \operatorname{supp}(\mu)$.

Proof. - Assume first that $M_{\alpha}\left(z_{0}\right)<\infty$ for some $z_{0} \in \mathbb{C} \backslash \operatorname{supp}(\mu)$. If we define the new measure

$$
\mathrm{d} \mu_{\alpha}(t)=(1+|t|)^{-\alpha} \mathrm{d} \mu(t)
$$

and let $\|\cdot\|_{*}$ denote the norm in $L^{\alpha}\left(\mu_{\alpha}\right)$, we have

$$
\left|p\left(z_{0}\right)\right| \leq M_{\alpha}\left(z_{0}\right)\|p\|_{*} \quad \text { for } p \in \mathbb{C}[t],
$$




\section{Christian Berg}

so by the Hahn-Banach Theorem there exists $g \in L^{\beta}\left(\mu_{\alpha}\right)$, where $\alpha$ and $\beta$ are dual exponents, such that

$$
p\left(z_{0}\right)=\int p(t) g(t) \mathrm{d} \mu_{\alpha}(t) \quad \text { for } p \in \mathbb{C}[t] .
$$

If this is applied to polynomials vanishing at $z_{0}$, we find for $p \in \mathbb{C}[t]$

$$
\int p(t) g(t)\left(t-z_{0}\right) \mathrm{d} \mu_{\alpha}(t)=0,
$$

and since $g(t)\left(t-z_{0}\right) /(1+|t|)^{\alpha} \in L^{\beta}(\mu)$, we see that $\mathbb{C}[t]$ is not dense in $L^{\alpha}(\mu)$.

Assume next that $\mathbb{C}[t]$ is not dense in $L^{\alpha}(\mu)$. Again by the Hahn-Banach Theorem there exists $g \in L^{\beta}(\mu) \backslash\{0\}$ such that

$$
\int p(t) g(t) \mathrm{d} \mu(t)=0 \quad \text { for } p \in \mathbb{C}[t] .
$$

The Cauchy transform

$$
\varphi(z)=\int \frac{g(t) \mathrm{d} \mu(t)}{t-z}
$$

is holomorphic in $\mathbb{C} \backslash \operatorname{supp}(\mu)$ and not identically zero. For $z_{0} \in \mathbb{C} \backslash \operatorname{supp}(\mu)$ and $p \in \mathbb{C}[t]$ we get by $(3.1)$

$$
\int \frac{g(t) p(t)}{t-z_{0}} \mathrm{~d} \mu(t)=p\left(z_{0}\right) \varphi\left(z_{0}\right)
$$

so if $\varphi\left(z_{0}\right) \neq 0$ we find

$$
\left|p\left(z_{0}\right)\right| \leq \frac{1}{\left|\varphi\left(z_{0}\right)\right|}\left(\int \frac{|p(t)|^{\alpha}}{\left|t-z_{0}\right|^{\alpha}} \mathrm{d} \mu(t)\right)^{1 / \alpha}\left(\int|g(t)|^{\beta} \mathrm{d} \mu(t)\right)^{1 / \beta},
$$

i.e.

$$
\left|p\left(z_{0}\right)\right| \leq K\left(z_{0}\right)\|g\|_{L^{\beta}(\mu)}\|p\|_{*},
$$

with

$$
K(z)=\frac{1}{|\varphi(z)|} \sup _{t \in \operatorname{supp}(\mu)} \frac{1+|t|}{|t-z|} .
$$

Note that $K$ is continuous in $\{z \in \mathbb{C} \backslash \operatorname{supp}(\mu) \mid \varphi(z) \neq 0\}$. 
If $\varphi\left(z_{0}\right)=0$ we choose $0<r<\operatorname{dist}\left(z_{0}, \operatorname{supp}(\mu)\right)$ so that $\varphi(z) \neq 0$ for $\left|z-z_{0}\right|=r$, and by the maximum principle we get

$$
\left|p\left(z_{0}\right)\right| \leq \max _{\left|z-z_{0}\right|=r}|p(z)| \leq K\|g\|_{L^{\beta}(\mu)}\|p\|_{*},
$$

with $K=\max _{\left|z-z_{0}\right|=r} K(z)$. The equations (3.2), (3.3) show that $M_{\alpha}\left(z_{0}\right)<\infty$.

Using the Lemma 3.7 Sodin [37] proved the following

Theorem 3.8. - Let $s>1$ and define

$$
\mu=\sum_{k=1}^{\infty} e^{-s k^{2} / 2} \delta_{e^{k}} .
$$

Then $\rho(\mu)=s$.

Remark. - By the above result any number $\lambda \in] 1, \infty[$ is the critical exponent of some measure $\mu \in \mathcal{M}^{*}(\mathbb{R})$. If $\mu$ is indeterminate and not an extreme point of $V_{\mu}$, then $\rho(\mu)=1$, and if the Carleman condition is fulfilled then $\rho(\mu)=\infty$. Thus any $\lambda \in[1, \infty]$ is the critical exponent of some $\mu \in \mathcal{M}^{*}(\mathbb{R})$. This result was established in [9] for $\lambda \leq 2$ and left open for $\lambda>2$, cf. [4].

\section{Rotation invariant measures}

The natural question whether Theorem 2.1 of Riesz extends to dimension $k>1$ was open for a number of years, cf. [23], [25, p. 529], until it was answered in the negative by Berg and Thill in [13]. In [25] the problem is ascribed to the physicist J. Challifour (1978).

THEOREM 4.1 ([13]). - For $k>1$ there exist determinate measures $\mu \in \mathcal{M}^{*}\left(\mathbb{R}^{k}\right)$ for which $\mathbb{C}[x]$ is not dense in $L^{2}(\mu)$.

In his survey paper [23] about the multidimensional moment problem Fuglede introduced two new notions of determinacy. The two notions were formulated as certain conditions of self-adjointness of the multiplication operators $X_{j} p=x_{j} p(x)$ with domain $\mathbb{C}\left[x_{1}, \ldots, x_{k}\right]$ in the Hilbert space $\mathcal{H}$ equal to the closure of $\mathbb{C}[x]$ in $L^{2}(\mu)$. 


\section{Christian Berg}

Fuglede characterized these notions in terms of approximation properties:

(i) $\mu$ is ultradeterminate if and only if $\mathbb{C}[x]$ is dense in $L^{2}\left(\left(1+\|x\|^{2}\right) \mathrm{d} \mu(x)\right)$.

(ii) $\mu$ is strongly determinate if and only if $\mathbb{C}[x]$ is dense in $L^{2}\left(\left(1+x_{j}^{2}\right) \mathrm{d} \mu\right)$ for $j=1, \ldots, k$.

(iii) If $\mu$ is strongly determinate, then $\mu$ is determinate and $\mathbb{C}[x]$ is dense in $L^{2}(\mu)$.

The condition of ultradeterminacy clearly implies strong determinacy. Schmüdgen [35] found an example of a strongly determinate measure which is not ultradeterminate and an example of a determinate measure which is not strongly determinate.

The latter also follows from Theorem 4.1, which is based on a study of rotation invariant measures in $\mathbb{R}^{k}$.

There is a bijection between rotation invariant measures $\mu$ on $\mathbb{R}^{k}$ and measures $\sigma$ on $[0, \infty$ [ established as $\sigma=\psi(\mu)$, the image measure of $\mu$ under $\psi: \mathbb{R}^{k} \rightarrow\left[0, \infty\left[\right.\right.$ defined by $\psi(x)=\|x\|^{2}$, i.e.

$$
\int_{0}^{\infty} f \mathrm{~d} \sigma=\int_{\mathbb{R}^{k}} f\left(\|x\|^{2}\right) \mathrm{d} \mu(x) .
$$

If $j:\left[0, \infty\left[\times S^{k-1} \rightarrow \mathbb{R}^{k}\right.\right.$ is given by $j(t, \xi)=\sqrt{t} \xi$, then $\mu=j(\sigma \otimes \omega)$, where $\omega$ is normalized surface measure on the unit sphere $S^{k-1}$. The moments of $\mu$ determine the moments of $\sigma$ and vice versa:

$$
\int_{\mathbb{R}^{k}} x_{1}^{n_{1}} \cdots x_{k}^{n_{k}} \mathrm{~d} \mu(x)=\int_{0}^{\infty} t^{(1 / 2) \Sigma n_{\imath}} \mathrm{d} \sigma(t) \int_{S^{k-1}} \xi_{1}^{n_{1}} \cdots \xi_{k}^{n_{k}} \mathrm{~d} \omega(\xi),
$$

which is zero unless all $n_{j}$ are even.

For $\sigma \in \mathcal{M}^{*}\left(\left[0, \infty\right.\right.$ [) (i.e. $\sigma \in \mathcal{M}^{*}(\mathbb{R})$ is supported by [0, [ ] there are two notions of determinacy to be distinguished: Determinacy in the sense of Stieltjes $(\operatorname{det}(\mathrm{S}))$ meaning that there is only one measure on $[0, \infty[$ with the same moments as $\sigma$, and (ordinary) determinacy (or determinacy in the sense of Hamburger, $\operatorname{det}(\mathrm{H})$ ), meaning that there is only one measure on $\mathbb{R}$ with the same moments as $\sigma$.

It should be emphasized that there exist measures $\sigma \in \mathcal{M}^{*}([0, \infty[)$ which are $\operatorname{det}(\mathrm{S})$ but indet $(\mathrm{H})$, i.e. in the infinite convex set $V_{\sigma}$ the measure 
$\sigma$ is the only one supported by [0, [. This measure $\sigma$ is necessarily $\sigma=\nu_{0}$, the $N$-extremal measure corresponding to the parameter $\varphi \equiv 0$ in the Nevanlinna parametrization, cf. [14], [21].

The paper [13] contains two key results relating $\mu \in \mathcal{M}_{\text {rot }}^{*}\left(\mathbb{R}^{k}\right)$ and the corresponding $\sigma \in \mathcal{M}^{*}([0, \infty[)$ such that (4.1) holds.

Theorem 4.2.- Given $\mu \in \mathcal{M}_{\text {rot }}^{*}\left(\mathbb{R}^{k}\right), k \geq 1$. Then $\mu$ is determinate if and only if $\sigma$ is $\operatorname{det}(S)$.

TheOREM 4.3.- Given $\mu \in \mathcal{M}_{\text {rot }}^{*}\left(\mathbb{R}^{k}\right), k>1$. Then $\mathbb{C}\left[x_{1}, \ldots, x_{k}\right]$ is dense in $L^{2}(\mu)$ if and only if $\mathbb{C}[t]$ is dense in $L^{2}\left(t^{p} \mathrm{~d} \sigma(t)\right)$ for $p=0,1, \ldots$

\section{Remarks}

(i) Theorem 4.3 requires $k>1$. For $k=1$ the corresponding assertion is (relating a symmetric measure $\mu$ and $\sigma=\psi(\mu)$ where $\psi(x)=x^{2}$ ): $\mathbb{C}[x]$ is dense in $L^{2}(\mu)$ if and only if $\mathbb{C}[t]$ is dense in $L^{2}(\sigma)$ and in $L^{2}(t \mathrm{~d} \sigma(t))$.

(ii) Theorem 4.3 is part of the following general result [13] about $\mu \in$ $\mathcal{M}^{*}\left(\mathbb{R}^{k}\right)$ not neccessarily rotation invariant and $\sigma=\psi(\mu)$ : Let $1 \leq \alpha<\infty$. If $\mathbb{C}[t]$ is dense in $L^{\alpha}\left(t^{(1 / 2) d \alpha} \mathrm{d} \sigma(t)\right)$ for $d=0,1, \ldots$, then $\mathbb{C}[x]$ is dense in $L^{\alpha}(\mu)$. As a consequence of this result we get the following relation between the critical exponents of $\mu$ and $\sigma$.

Corollary 4.4. - Let $\mu \in \mathcal{M}^{*}\left(\mathbb{R}^{k}\right)$ and $\sigma=\psi(\mu)$. If $1<\rho(\sigma)$ then $\rho(\sigma) \leq \rho(\mu)$.

Proof. - Assume that $\mathbb{C}[t]$ is dense in $L^{\alpha}(\sigma)$, where $1<\alpha<\infty$. It suffices to prove that $\mathbb{C}[t]$ is dense in $L^{\alpha^{\prime}}\left(t^{(1 / 2) d \alpha^{\prime}} \mathrm{d} \sigma(t)\right)$ for $d=0,1, \ldots$ and $1<\alpha^{\prime}<\alpha$. For $p \in \mathbb{C}[t]$ and $f \in C_{c}([0, \infty[)$ we find by Hölder's inequality

$$
\begin{aligned}
& \int|f(t)-p(t)|^{\alpha^{\prime}} t^{(1 / 2) d \alpha^{\prime}} \mathrm{d} \sigma(t) \leq \\
& \leq\left(\int|f(t)-p(t)|^{\alpha} \mathrm{d} \sigma(t)\right)^{\alpha^{\prime} / \alpha}\left(\int t^{(1 / 2) d \alpha^{\prime} s} \mathrm{~d} \sigma(t)\right)^{1 / s},
\end{aligned}
$$

where $s$ is the dual exponent of $\alpha / \alpha^{\prime}$. The first factor can be made as small as we please by assumption.

Combining Theorems 4.2 and 4.3 we get the following result. 
THEOREM 4.5. - Let $\mu \in \mathcal{M}_{\text {rot }}^{*}\left(\mathbb{E}^{k}\right), k>1$ with corresponding $\sigma \in$ $\mathcal{M}^{*}\left(\left[0, \infty[)\right.\right.$. Then $\mu$ is determinate and $\mathbb{C}[x]$ is not dense in $L^{2}(\mu)$ if and only if $\sigma$ is $\operatorname{det}(S)$ and there exists $p \in \mathbb{N}$ such that $\mathbb{C}[t]$ is not dense in $L^{2}\left(t^{p} \mathrm{~d} \sigma(t)\right)$.

The measures $\sigma$ in the theorem above are necessarily obtained from some $N$-extremal measure $\tau \in \mathcal{M}^{*}([0, \infty[)$ by removing a finite number of its masses (cf. sect. 5). In particular $\mu$ has the form

$$
\mu=\sum_{n=0}^{\infty} \alpha_{n} \omega_{\sqrt{r_{n}}}, \quad \alpha_{n}>0, \quad 0 \leq r_{0}<r_{1}<\ldots \rightarrow \infty,
$$

where $\omega_{r}$ is the normalized uniform distribution on the sphere $\|x\|=r$.

CoRollary 4.6. - If $\mu \in \mathcal{M}_{\mathrm{rot}}^{*}\left(\mathbb{R}^{k}\right)$ is determinate and absolutely continuous, then $\mathbb{C}[x]$ is dense in $L^{2}(\mu)$.

Example. - Let $\nu_{0}$ be indet(S) and the $N$-extremal solution corresponding to the parameter value $\varphi \equiv 0$. The measure $\sigma=\nu_{0}-\nu_{0}(\{0\}) \delta_{0}$ has the properties (cf. [13]): $\sigma$ is $\operatorname{det}(\mathrm{H})$, and $\mathbb{C}[t]$ is dense in $L^{2}\left(t^{p} \mathrm{~d} \sigma(t)\right)$ for $p=0,1,2$ but not for $p \geq 3$. The corresponding rotation invariant measure

$$
\mu=\sum_{n=1}^{\infty} \alpha_{n} \omega \sqrt{r_{n}}, \quad \text { where } \sigma=\sum_{n=1}^{\infty} \alpha_{n} \delta_{r_{n}},
$$

has the following properties (cf. [13], [7]):

(i) $\mu$ is determinate.

(ii) $\mathbb{C}[x]$ is dense in $L^{\alpha}(\mu)$ for $1 \leq \alpha<2$ but not for $\alpha=2$.

(iii) $\operatorname{dim}\left(L^{2}(\mu) \ominus \overline{\mathbb{C}[x]}\right)=\infty$.

(iv) The marginal distributions are indeterminate.

An explicit example of a measure $\nu_{0}$ as above is given in section 6 .

\section{Measures of finite index}

Inspired by Theorem 4.5 the following (Stieltjes) index of determinacy was introduced in [13] for measures $\sigma \in \mathcal{M}^{*}([0, \infty[)$ which are $\operatorname{det}(\mathrm{S})$ :

$$
i(\sigma)=\sup \left\{k \in \mathbb{N}_{0} \mid t^{k} \mathrm{~d} \sigma(t) \text { is } \operatorname{det}(\mathrm{S})\right\},
$$

and the following result was obtained. 
Theorem 5.1.- For $\sigma \in \mathcal{M}^{*}([0, \infty[)$ which is $\operatorname{det}(S)$ the following conditions are equivalent:

(i) $i(\sigma)=0$,

(ii) $\sigma=\nu_{0}-\nu_{0}(\{0\}) \delta_{0}$, where $\nu_{0}$ is the $N$-extremal solution with parameter value 0 corresponding to a Stieltjes moment problem which is indet $(S)$.

Furthermore, the measure $\sigma$ has finite index $j \geq 1$ if and only if $\sigma=\alpha \delta_{0}+t^{-j} \mathrm{~d} \tau(t)$, where $\tau \in \mathcal{M}^{*}([0, \infty[)$ has index zero and $\alpha \geq 0$.

Motivated by the above and polynomials orthogonal with respect to a matrix of measures, Berg and Duran [10] introduced the following index of determinacy for determinate measures $\mu \in \mathcal{M}^{*}(\mathbb{R})$ and $z \in \mathbb{C}$

$$
\operatorname{ind}_{z}(\mu)=\sup \left\{k \in \mathbb{N}_{0}|| t-\left.z\right|^{2 k} \mathrm{~d} \mu(t) \text { is } \operatorname{det}(\mathrm{H})\right\},
$$

and the following result was proved:

THEOREM 5.2

(i) If $\tau=\sum_{\lambda \in \Lambda} a_{\lambda} \delta_{\lambda}$ is indeterminate and $N$-extremal, and if $\Lambda_{0} \subset \Lambda$ has $k+1$ points, then for $\mu=\sum_{\lambda \in \Lambda \backslash \Lambda_{0}} a_{\lambda} \delta_{\lambda}$ we have

$$
\operatorname{ind}_{z}(\mu)= \begin{cases}k & \text { for } z \notin \operatorname{supp}(\mu) \\ k+1 & \text { for } z \in \operatorname{supp}(\mu) .\end{cases}
$$

(ii) If $\mu$ is determinate and $\operatorname{ind}_{z}(\mu)<\infty$ for some $z \in \mathbb{C}$, then $\mu$ is derived from an $N$-extremal measure $\tau$ as above and $\operatorname{ind}_{z}(\mu)$ is given by (5.3).

Let $\mu \in \mathcal{M}^{*}(\mathbb{R})$ be determinate. We define ind $(\mu)=k$ if (5.3) holds; otherwise $\operatorname{ind}_{z}(\mu)=\infty$ for all $z \in \mathbb{C}$ and we put ind $(\mu)=\infty$.

If $\sigma \in \mathcal{M}^{*}([0, \infty[)$ is $\operatorname{det}(\mathrm{H})$ and a fortiori $\operatorname{det}(\mathrm{S})$, the following relation holds between the two indices of determinacy:

$$
\operatorname{ind}_{0}(\sigma)=\left[\frac{1}{2} i(\sigma)\right]
$$

where $[x]$ is the integral part of $x$ in case $x<\infty$ and $[\infty]=\infty$. 
A determinate measure $\mu \in \mathcal{M}^{*}(\mathbb{R})$ of finite index is discrete and can be written as

$$
\mu=\sum_{n=1}^{\infty} a_{n} \delta_{\lambda_{n}}
$$

where $a_{n}>0$ and $0 \leq\left|\lambda_{1}\right| \leq\left|\lambda_{2}\right| \leq \ldots$. The canonical product

$$
F_{\mu}(z)=\lim _{N \rightarrow \infty} \prod_{n=1}^{N}\left(1-\frac{z}{\lambda_{n}}\right)
$$

(with $1-z / \lambda_{1}$ replaced by $z$ if $\lambda_{1}=0$ ) converges uniformly on compact subsets of $\mathbb{C}$ to an entire function of minimal exponential type vanishing at $\Lambda=\left\{\lambda_{n}\right\}=\operatorname{supp}(\mu)$, cf. [10].

Let $\left(P_{n}\right)$ be the corresponding orthonormal polynomials. The result of Hamburger in section 2 can be stated that $\left(P_{n}(z)\right) \in \ell^{2}$ if and only if $F_{\mu}(z)=0$. In [10] we considered the question whether $\left(P_{n}^{(m)}(z)\right)_{n} \in \ell^{2}$, and it turned out that for $\operatorname{ind}(\mu)>0, m \geq 1$ this is never the case, but for $\operatorname{ind}(\mu)=0$ it was established that $\left(P_{n}^{(m)}(z)\right)_{n} \in \ell^{2}$ if and only if $F_{\mu}^{(m)}(z)=0$, and this holds for countably many points.

In [11] we considered general discrete differential operators of the form

$$
T=\sum_{\ell=1}^{N} \sum_{j=0}^{k_{\ell}} a_{\ell, j} \delta_{z_{\ell}}^{(j)},
$$

where $k_{\ell} \geq 0, a_{\ell, j} \in \mathbb{C}$, and $z_{1}, \ldots, z_{N}$ are different complex numbers. We are interested in the question whether $\left(T\left(P_{n}\right)\right) \in \ell^{2}$, which is of interest because this condition is equivalent to the assertion that

$$
p \mapsto T(p)=\sum_{\ell=1}^{N} \sum_{j=0}^{k_{\ell}} a_{\ell, j} p^{(j)}\left(z_{\ell}\right)
$$

has a continuous linear extension from $\mathbb{C}[t]$ to $L^{2}(\mu)$.

THEOREM 5.3.- Suppose $\operatorname{ind}(\mu)<\infty$ and let $F_{\mu}$ be as above. Then $\left(T\left(P_{n}\right)\right)_{n} \in \ell^{2}$ if and only if $T\left(z^{k} F_{\mu}(z)\right)=0$ for $k=0,1, \ldots, \operatorname{ind}(\mu)$.

If $z_{1}, \ldots, z_{N} \in \mathbb{C} \backslash \operatorname{supp}(\mu)$ and $k_{1}, \ldots, k_{N} \geq 0$ are fixed but $a_{\ell, j} \in \mathbb{C}$ variable then

$$
\begin{aligned}
\operatorname{dim}\left\{T \mid\left(T\left(P_{n}\right)\right)_{n} \in \ell^{2}\right\} & =\left(\sum_{\ell=1}^{N}\left(k_{\ell}+1\right)-\operatorname{ind}(\mu)-1\right)^{+} . \\
& -28-
\end{aligned}
$$


This result can be used to realize $L^{2}(\mu)$ as a Hilbert space of entire functions in the sense of de Branges [16] in case ind $(\mu)<\infty$. For details see [12].

Determinate measures of finite index are in a certain sense indeterminate measures which are canonical of negative order. This is to be understood in the following sense: If $\mu$ is indeterminate and canonical of order $n \geq 0$, and if $0 \leq k$ masses are removed, then the modified measure $\tilde{\mu}$ is canonical of order $n-k$ if $k \leq n$. However if $k>n$, then the modified measure $\tilde{\mu}$ is determinate and $\operatorname{ind}(\tilde{\mu})=k-n-1$.

The analogy can be pursued further in the direction of Theorem 2.2. It turns out that for determinate measures $\mu$ of finite index $k$, not only the polynomials are dense in $L^{2}(\mu)$, but also a certain polynomial ideal of co-dimension $k+1$ in $\mathbb{C}[x]$ is dense in $L^{2}(\mu)$ :

Theorem $5.4([11])$. - Assume that ind $(\mu)=k$ and let $R \in \mathbb{C}[x]$ be a polynomial of degree $k+1$ such that $R(z) \neq 0$ for $z \in \operatorname{supp}(\mu)$. Then the ideal $R \mathbb{C}[x]$ is dense in $L^{2}(\mu)$.

\section{Examples}

Let $0<q<1$ be fixed. We define

$$
(z ; q)_{n}=\prod_{j=1}^{n}\left(1-z q^{j-1}\right), \quad n=0,1, \ldots, \infty, z \in \mathbb{C},
$$

and shall also use the short notation

$$
\left(z_{1}, \ldots, z_{k} ; q\right)_{n}=\prod_{j=1}^{k}\left(z_{j} ; q\right)_{n}
$$

Let

$$
G_{n}(a)=\sum_{k=0}^{n}\left[\begin{array}{l}
n \\
k
\end{array}\right]_{q} q^{k(k-n)} a^{k}
$$

where

$$
\left[\begin{array}{l}
n \\
k
\end{array}\right]_{q}=\frac{(q ; q)_{n}}{(q ; q)_{k}(q ; q)_{n-k}}, \quad 0 \leq k \leq n
$$

is the Gauss binomial coefficient. 


\section{Christian Berg}

Al-Salam and Carlitz [2] noticed that

$$
s_{n}(a)=\sum_{k=0}^{n}\left(\begin{array}{l}
n \\
k
\end{array}\right)(-1)^{n-k} G_{k}(a)
$$

is a Stieltjes moment sequence for $0<a$ and found a measure with these moments

$$
\begin{aligned}
m^{(a)} & =(a q ; q)_{\infty} \sum_{n=0}^{\infty} \frac{a^{n} q^{n^{2}}}{(a q, q ; q)_{n}} \delta_{\left(q^{-n}-1\right)}, \quad \text { when } 0<a<\frac{1}{q} \\
\sigma^{(a)} & =(q / a ; q)_{\infty} \sum_{n=0}^{\infty} \frac{a^{-n} q^{n^{2}}}{(q / a, q ; q)_{n}} \delta_{\left(a q^{-n}-1\right)}, \quad \text { when } q<a .
\end{aligned}
$$

These measures were considered further by Chihara ([20], [21]), who proved that the moment problem is indeterminate as a Hamburger moment problem for $q<a<1 / q$ and otherwise determinate. Furthermore $m^{(a)}$ is the $N$-extremal measure corresponding to the parameter value $t=0$ when $q<a<1 / q$. Ismail [26] found the right normalization of the measures $m^{(a)}$ and $\sigma^{(a)}$. For a self-contained treatment of this moment problem we refer to the paper [15] by Berg and Valent. It also contains an explicit determination of the functions $A, B, C, D$ in the indeterminate case. In addition it is shown that Carleman's series $\sum 1 / \sqrt[2 n]{s_{2 n}(a)}$ converges for $0<a .0<q<1$. (This is a concrete example demonstrating that Carleman's condition is not necessary for determinacy.)

For further examples of $N$-extremal measures see [15] and [27].

\section{References}

[1] Akhiezer (N. I.) . - The classical moment problem, Oliver and Boyd, Edinburgh 1965.

[2] Al-Salam (W. A.) and Carlitz (L.) .- Some orthogonal q-polynomials. Math. Nachr., 30 (1965), pp. 47-61.

[3] Baillaud (B.) and Bourget (H.) .- Correspondance d'Hermite et de Stieltjes I, II, Gauthier-Villars, Paris 1905.

[4] Berg (C.) .- Critical exponents for measures. in: Open problems, ed. W. Van Assche, J. Comput. Appl. Math.. 48 (1993), p. 227.

[5] BERG (C.) .- $L^{2}$-approximation with respect to rotation invariant measures, Suppl. Rend. Circ. Mat. Palermo. Serie II, 33 (1993). pp. 211-217. 
[6] BERG (C.) .- Indeterminate moment problems and the theory of entire functions, J. Comput. Appl. Math., 65 (1995), pp. 27-55.

[7] Berg (C.) .- Recent results about moment problems, in: Probability measures on groups and related structures XI, World Scientific, Singapore 1995.

[8] Berg (C.) and Christensen (J. P. R.) .- Density questions in the classical theory of moments, Ann. Inst. Fourier, 31, $\mathrm{n}^{\circ} 3$ (1981), pp. 99-114.

[9] Berg (C.) and Christensen (J. P. R.) .- Exposants critiques dans le problème des moments, C.R. Acad. Sci. Paris Série I, 296 (1983), pp. 661-663.

[10] Berg (C.) and Duran (A. J.) .- The index of determinacy for measures and the $\ell^{2}$-norm of orthonormal polynomials, Trans. Amer. Math. Soc., 347 (1995), pp. 2795-2811.

[11] Berg (C.) and Duran (A. J.) .- When does a discrete differential perturbation of a sequence of orthonormal polynomials belong to $\ell^{2}$ ? J. Funct. Anal., 136 (1996), pp. 127-153.

[12] Berg (C) and Duran (A. J.) . - Orthogonal polynomials, $L^{2}$-spaces and entire functions, Math. Scand. (to appear).

[13] Berg (C.) and Thill (M.) .- Rotation invariant moment problems, Acta Math. 167 (1991), pp. 207-227.

[14] Berg (C.) and Thill (M.) .- A density index for the Stieltjes moment problem, in: Orthogonal Polynomials and their Applications, ed. C. Brezinski, L. Gori and A. Ronveaux, IMACS Annals on computing and applied mathematics, 9 (1991), pp. 185-188.

[15] Berg (C.) and VALENT (G.) .- The Nevanlinna parametrization for some indeterminate Stieltjes moment problems associated with birth and death processes, Methods and Applications of Analysis, 1 (1994), pp. 169-209.

[16] De Branges (L.) .- Hilbert spaces of entire functions, Prentice-Hall, Englewood Cliffs, N.J.. 1968.

[17] Buchwalter (H.) and Cassier (G.) .- Mesures canoniques dans le problème classique des moments, Ann. Inst. Fourier, 34 (1984), pp. 45-52.

[18] CASSier (G.) .- Mesures canoniques dans le problème classique des moments, C.R. Acad. Sci., 296, Série I (1984), pp. 717-719.

[19] CAssier (G.) .- Problème des moments sur un compact de $\mathbb{R}^{n}$ et représentation de polynômes à plusieurs variables, J. Funct. Analysis, 58 (1984), pp. 254-266.

[20] Chinara (T. S.) .- On indeterminate Hamburger moment problems, Pac. J. Math., 27 (1968), pp. 475-484.

[21] Chimara (T. S.) .- Indeterminate symmetric moment problems, Math. Anal. Appl., 85 (1982), pp. 331-346.

[22] Choquet (G.) .- Le problème des moments, Séminaire d'initiation à l'analyse (1962), Paris.

[23] Fuglede (B.) . - The multidimensional moment problem, Expo. Math., 1 (1983), pp. $47-65$.

[24] Hamburger (H.) .- Über eine Erweiterung des Stieltjesschen Momentenproblems, Math. Ann., 81 (1920), pp. 235-319.; 82 (1921), pp. 120-164. 168-187. 
[25] Havin (V. P.) et al. (editors) . - Linear and complex analysis problem book. 199 research problems, Lecture Notes in Mathematics, 1043, Springer, Berlin Heidelberg - New York 1984.

[26] Ismail (M. E. H.) - - A queueing model and a set of orthogonal polynomials, J. Math. Anal. Appl., 108 (1985), pp. 575-594.

[27] Ismail (M. E. H.) and MAsson (D. R.) .- Q-Hermite polynomials, biorthogonal rational functions and Q-beta integrals, Trans. Amer. Math. Soc., 346 (1994), pp. 63-116.

[28] KJELDSEN (T. H.) . - The early history of the moment problem, Historia Math.. 20 (1993), pp. 19-44.

[29] Koosis (P.) .- The logarithmic integral I, Cambridge University Press, Cambridge 1988.

[30] NAIMARK (M. A.) - - Extremal spectral functions of a symmetric operator, Izv. Akad. Nauk. SSSR ser. matem., 11 (1947), pp. 327-344; Dokl. Akad. Nauk. SSSR, 54 (1946), pp. 7-9.

[31] Nevanlinna (R.) .- Asymptotische Entwicklungen beschränkter Funktionen und das Stieltjessche Momentenproblem, Ann. Acad. Scient. Fennicae, (A), 18, $\mathrm{n}^{\circ} 5$ (1922), pp. 1-53.

[32] Petersen (L. C.) .- On the relation between the multidimensional moment problem and the one-dimensional moment problem, Math. Scand., 51 (1982), pp. 361-366.

[33] Riesz (M.) . - Sur le problème des moment. Troisième Note, Arkiv för matematik, astronomi och fysik, 17, $\mathrm{n}^{\circ} 16$ (1923), pp. 1-52.

[34] Riesz (M.) .- Sur le problème des moments et le théorème de Parseval correspondant, Acta Litt. Ac. Sci. Szeged, 1 (1923), pp. 209-225.

[35] SCHMÜDGEN (K.) - - On determinacy notions for the two dimensional moment problem, Ark. Mat., 29 (1991), pp. 277-284.

[36] SCHMÜDGEN (K.) . - The K-moment problem for compact semi-algebraic sets, Math. Ann., 289 (1991), pp. 203-206.

[37] Sodin (K.) .- A note on the Hall-Mergelyan theme, Mathematical Physics, Analysis and Geometry (To appear).

[38] Stielt jes (T. J.) .- Recherches sur les fractions continues, Annales de la Faculté des Sciences de Toulouse, 8 (1894), pp. 1-122; 9 (1895), pp. 5-47. 